\title{
VANTAGENS DO ENSINO NO LABORATÓRIO DE ENFERMAGEM
}

Marıa Romana Friedlander*

FRIEDLANDER, M. R. Vantagens do ensino no laboratório de enfermagem. Rev. Esc. Enf. USP., v.28, n.2, p. 227-33, ago. 1994.

Revendo a literatura, a autora descreve algumas vantagens do ensino das habilidades perceptivo-motomas (ou psicomotoras) no laboratório de enfermagem como treinamento anterior ao estágio em campo clínico. Os argumentos utilizados são de ordem ética (levantando a questāo do erro cometido pelo aluno no cliente), de natureza psicológica, apontando os conflitos do aluno, do cliente e do professor, aspectos relacionados ao custo-beneficio desse treinamento preliminar e de ordem pedagógica. Estes ultimos aspectos apolam-se nas teorias da aprendizagem perceptivo-motora que confirmam a importância do ensino no laboratório de enfermagem.

UNITERMOS: Educaçāo em enfermagem. Ensino.

A crescente evoluçāo do pensamento e do conhecimento de enfermagem tem pressionado os educadores a adicionarem cada vez maior número de novos temas aos currículos de enfermagem. Essa adiçāo tem sido realizada em detrimento das habilidades técnicas provocando insatisfaçōes no pessoal da assistência e nos próprios educadores (GOMEZ; GOMEZ, 1984). Essa insatisfação estimula a reflexão sobre todo o processo ensino-aprendizado, especificamente, das habilidades eminentemente técnicas ou psicomotoras.

Revendo a literatura, desta última década, conclui-se que podem ser apontados argumentos de natureza ética, psicológica, econômica e pedagógica para defender o uso do laboratório de enfermagem no que concerne à aprendizagem inicial e ao desenvolvimento do estudante frente às habilidades técnicas de enfermagem. ELLIOTT et al(1982), em um estudo sobre a ênfase que merecem as habilidades psicomotoras nos curriculos das escolas de enfermagem dos Estados Unidos e Canadá, constatam que “Os resultados obtidos apoiam a tese de que o laboratorio de enfermagem é quase universalmente usado para o ensino e a aprendizagem das habilidades psicomotoras".

- Enfermeira. Professora Adjunta do Departamento de Enfermagem da Escola Paulista de Medicina. 
Um dos mais influentes argumentos tem sido a crescente valorização de toda a sociedade e, em especial, dos profissionais da área da saúde em relação ao direito do paciente em receber uma assistência eficiente e segura. $O$ número de processos legais que têm atingido os hospitais e os profissionais e o crescimento da rede de seguros sociais vem demonstrando a valorização da segurança na assistência à saúde. A enfermagem neste contexto está sendo levada a discutir e a preocupar-se com a segurança do serviço que presta e os educadores debatem essa segurança na aprendizagem de enfermagem (CORCORAN, 1977).

O erro é premissa do que não sabe, do que está na situação de aprendiz. Mas no caso da aprendizagem junto ao cliente, o erro não pode ser admitido. As consequiências podem ser graves e irreparáveis tanto para o cliente como para o estudante e as instituiçōes envolvidas. Erros, como trocas de medicamentos, inadequadas conexōes de tubos ou observaçōes mal interpretadas. cometidos por estudantes, seriam da responsabilidade de quem? Do estudante que está a aprender e, portanto, não sabe? Da instituição que permite "os que não sabem" aí prestarem serviços? Das escolas de enfermagem que permite que um professor supervisione, em campo, 12 alunos iniciantes? Do professor que se responsabiliza por orientar e supervisionar um número de estudantes acima da sua capacidade em os atender?

O cliente, quando procura um serviço çe assistência espera um alto nivel de conhecimento, de experiéncia, de segurar. a e de conforto. Procura e espera a eficiência e tem direito a ela (TAYLOR; CLEVELAND, 1984).

INFANTE $(1981,1985)$ chama a atenção para a responsabilidade do professor em assegurar, antes da entrada do estudante no setor clínico, o melhor e o maior desenvolvimento possivel no que concerne à execução das habilidades práticas. Afirma que uma aprendizagem cuidadosa em laboratório permite o alcance de um bom desenvolvimento porque torna o aluno mais seguro em sua "performance". Prestar assistência de enfermagem não é sinônimo de aprender a prestar essa assistência (INFANTE, 1981; FRIEDLANDER, 1984).

Outro fator que está a estimular os professores em direção ao laboratório de enfermagem é a constatação do nível de ansiedade e de frustração apresentado por muitos estudantes quando enfrentam seus primeiros clientes (BOYD et al, 1983; BUTHERFIELD, 1983; TAYLOR; CLEVELAND, 1984; FRIEDLANDER, 1984; PAGANA, 1988; BELL, 1991; BLENNER, 1991).

$O$ não conhecimento adicionado à condiçāo de não poder errar produz conflitos sérios que dificultam a aprendizagem. O estudante pressionado pela situaçāo frente a um cliente, onde nāo lhe é permitido o engano, sein se sentir seguro e confiante em sua própria habilidade, e, como único auxílio, tem um professor que também deve dar atençāo a outros, pode desenvolver um estado de tensão ansiosa que ocasiona situações difíceis.

No caso das primeiras experiências não é raro o fato de estudantes se sentirem completamente bloqueados, quase incapacitados de falar e agir, apresentarem sudorese e palidez intensas, não poderem decidir ou tomarem qualquer iniciativa. A situação torna-se extremamente estressante, agindo 
como reforço negativo e, conseqüentemente, provocar o desinteresse, o desestímulo, o afastamento ou 0 medo em enfrentar a relação aluno-paciente. $O$ professor pode transmitir um pouco de segurança e estímulo mas, como está freqüentemente a dar atenção a muitos outros estudantes, o seu efeito é quase minimo. $\mathrm{E}$ as experiências de aprendizagem vão acontecendo de maneira muito lenta com grande perda de tempo.

TAYLOR; CLEVELAND (1984), com base em experiència prática realizada, defendem a utilização criativa e bem planejada do laboratório de enfermagem, num momento anterior à prática clínica, para diminuir essa ansiedade.

FRIEDLANDER (1984) verificou que a maioria dos estudantes $(94,7 \%$ ) “concorda que a prática prévia no Laboratório de Enfermagem guiada por um roteiro auto-instrucional permite que o aluno vá para junto do cliente com maior segurança". Essa constatação foi confirmada, em 1993, pela mesma autora (FRIEDLANDER;.LUACUTE, 1993c).

Contudo, o papel que exerce o treinamento prévio do estudante em laboratório de enfermagem ainda deve ser estudado com maior acuidade pois os resultados das pesquisas são contraditórios. Um estudo levado a efeito por BOYD et al (1983), relacionado à execução da injeção intramuscular, não encontrou diferenças significativas entre 08 grupos de estudantes que praticaram antecipadamente no laboratório de enfermagem e os grupos que não desenvolveram essa prática. Da mesma forma, MEGEL et al (1987) constataram não haver diferença no relato da ansiedade na situação simulada quando comparado à situação real (hospital).

Ainda sob o ponto de vista psicológico, a frustraçāo, entre os professores, quando têm que enfrentar a prática clínica com um grande número de estudantes iniciantes, está aparecendo descrita na literatura. O professor sente-se incapaz de prover um ensino adequado, dinâmico, à altura das perspectivas e necessidades dos estudantes e, ao mesmo tempo, que possa garantir a segurança e a eficiência ao cliente. $O$ conflito entre ser enfermeiro e ter como o mais al to valor o bem estar do cliente e ser professor responsável pelo desenvolvimento de estudantes, instala-se e, frequentemente, arrasta consigo a frustração. O professor sente que nunca está fazendo algo bem feito (TAYLOR; CLEVELAND, 1984).

Além dos aspectos de ordem ética e psicológica já citados, podemos discernir aspectos econômicos que estão exercendo importante influência nas decisões educacionais a favor da utilização mais criteriosa do laboratório de enfermagem. São os referentes ao alto custo da prática clínica e suas consequências no ensino da enfermagem.

$O$ ensino em campo clínico exige uma baixa proporção de alunos por professor. A segurança do cliente, o nivel de aprendizagem, o tamanho das unidades de internação e o número de clientes internados impedem que cada professor tome sob sua responsabilidade um grande número de estudantes, de uma só vez (TAYLOR; CLEVELAND, 1984; GOTHLER, 1985).

$O$ custo da prática clínica também aumenta se se considerar que $o$ desperdício de material descartável e esterilizado é grande quando esse 
material é manipulado por estudantes. A inabilidade própria do estudante e a necessidade da repetição podem influir de forma significativa no custo da prática clínica.

GOTHLER (1985) constata que, nos Estados Unidos, as escolas estão dando prioridade à contratação de professores em meio-período com a única responsabilidade de supervisionar os estudantes nos estágios clínicos: critica esta soluçāo porque a comunicação e a coordenação dos programas levados a efeito por professores com diferentes tipos de contratos de trabalhos traz inúmeros problemas. Cita também programas que utilizam estudantes de classes mais adiantadas para ensinar as mesmas habilidades numa tentativa de reduzir os custos operacionais do ensino da enfermagem.

Outro reflexo dos fatores econômicos na prática clínica, levantado pela mesma autora, é que, com freqüência os estágios clínicos são orientados para a realização de trabalho em detrimento da aprendizagem.

De fato é uma realidade, à qual não se pode fechar os olhos, que ao implementar o aprender-fazendo de Dewey se corre o risco de fazer sem aprender. Se o ensino clínico nāo for bem planejado, bem orientado, bem supervisionado e bem avaliado pode tornar-se um campo de repetiçōes desnecessárias e de experiências sem significado pedagógico com um aproveitamento mínimo para os estudantes. Não são poucas as situaçōes em que se constata a transformação do aprendiz de enfermagem em mão-de-obra barata dos serviços de assistência. E não foram poucas as escolas que se fundaram com a finalidade subjacente de se obter o barateamento do custo da assistência ao cliente.

Também, sob este aspecto, um maior número de pesquisas deve ser realizado pois os resultados têm sido contraditorios. Enquanto HALLAL; WELSH ( 1984) e FRIEDLANDER (1984) afirmam, com base em suas proprias experiências, que o laboratório de enfermagem é um recurso de aprendizagem eficiente sob o ponto de vista financeiro; HEGSTAD; ZSOHAR (1986) concluiram que a prática em laboratório de enfermagem é cara e não produz efeitos no conhecimento do estudante que a justifique.

As investigações que têm como objetivo o estudo da relação custo-benefício trabalham com variáveis que sofrem a influência direta de condiçōes locais e temporais. Portanto, os ispectos econômicos e sua interferência precisam ser considerados cuidadosamente ao se planejarem as estratégias de ensino, lembrando que, nos dias atuais podemos contar com o auxilio: das fitas de vídeo (ARBUES-MOREIRA; FRIEDLANDER, 1992: BALDWIN et al 1991) ensino por computador (HODSON et al, 1988; BLENNER, 1991) e dispositivos simplificados (NASCIMENTO; CANÇADO, 1991).

Outro aspecto sob o qual a prática no laboratório deve ser analisada e que se considera de extrema importância, é o que se relaciona aos aspectos da aprendizagem propriamente dita.

BUTHERFIELD (1983) defende a utilização do laboratório de enfermagem com base no referencial teórico de Poulton. Esse modelo explica como o cérebro humano trabalha durante a execução de uma habilidade. Esse autor sugere a aquisição da habilidade como um processo contínuo no qual os 
estímulos são interiorizados por meio dos sentidos e exteriorizados por meio do trabalho muscular. Intermediando essas duas fases dos extremos do processo dá-se a atividade cerebral constituída por: trabalho da memória, seleção dos estímulos de entrada, processamento e seleção dos comportamentos de saída. A memória de longa duração pode interferir tanto nos estímulos de entrada como nos estímulos de saída.

Com base nesse modelo, é explicado como o "stress" e a fadiga podem dificultar ou impedir a qualidade da decisão apoiando a idéia de que esse "stress" pode ter efeitos cumulativos. Contudo, nos movimentos altamente automatizados, segundo a mesma autora, o efeito do cansaço faz-se sentir com menor intensidade. Uma alta carga nos estímulos sensoriais, como acontece na situaçāo clínica, pode dificultar a aprendizagem das habilidades. $O$ estudante deve poder concentrar-se em movimentos especificos sem que o sistema de entrada seja solicitado por outros estímulos fortes como o ruído e o movimento. HALLAL; WELSH (1984) também constataram a dificuldade do estudante em concentrar sua atenção nas atividades de aprendizagem no setor clínico devido à constante estimulaçāo exercida por esse setor.

FRIEDLANDER (1993-b) confirma que só após o estudante ter dominado as habilidades básicas motoras de enfermagem é que ele se encontra pronto para desenvolver as habilidades intelectuais e prover uma melhor assistência no setor clínico.

O grau de automatização que se pode obter com um treinamento em laboratório (situação simulada), anterior à prática de campo, poderá bloquear o efeito negativo da super-estimulação da área clínica.

Ainda sob o ponto de vista pedagógico sabe-se que a prática em laboratório de enfermagem provê uma boa destreza manual (BOYD et al, 1983) e permite repetiçōes que a situação que envolve clientes torna inseguras e impraticáveis (HEGSTAD; ZSOHAR, 1986) comprometendo o bom aproveitamento do estudante. Esse aproveitamento, bem como todo o processo ensino-aprendizagem, pode ser controlado com maior facilidade em situação simulada e a precisảo desse controle pode oferecer subsídios para uma melhor avaliação da prática clínica dos estudantes.

\section{CONCLUSĀO}

Pelo aqui exposto, conclui-se que o laboratório de enfermagem e as atividades de aprendizagem nele desenvolvidas podem apresentar uma série de vantagens. Deve, portanto, ser tido como um recurso instrucional potencialmente válido, como têm apontado alguns pesquisadores em estudos abordando vários aspectos (McDONALD, 1987), inclusive a metodologia de ensino mais apropriada (BALDWIN et al, 1991; BELL, 1991; FRIEDLANDER; SOARES, 1993-b; FRIEDLANDER; LUACUTE, 1993-c) e a sua organização (HAYASHIDA, 1992).

Por outro lado, deixa-se claro que não pode ser considerado como uma alternativa substitutiva da prática clínica. A prática clínica não pode ser 
substituída pois há aspectos da aprendizagem que só podem ser desenvolvidos na relação aluno-paciente.

O laboratório deve ser usado como um recurso de apoio e de auxílio à prática clínica, um recurso que melhore as condições da aprendizagem junto aos clientes, uma estratégia que impeça algumas distorçōes dessa aprendizagem.

Contudo, para esclarecer muitos dos aspectos aqui mencionados, verifica-se uma grande falta de investigaçōes e estudo nesta área da prática da aprendizagem, as quais poderiam com maior segurança orientar o professor na decisão dos recursos a utilizar.

FRIEDLANDER, M. R. The advantages of the nursing laboratory teaching. Rev. Esc. Enf. USP., v.28, n.2, p. 227-33, aug. 1994.

Reviewing the literatur, the author describes some advantages of the psychomotor skill teaching at nursing laboratory as a previous training to the clinical service setting. The arguments herein presented are related, first to the ethics (questioning the mistakes mader by the trainee on the patient); second, to the psychological nature by pointing out the trainee, the patient and the teacher's conflicts, as well as aspects on the cost-benefit obtained with this preliminary training; and third, to the pedagogy. These last mentioned aspects are supported on the psychomotor learning theories which confirm the importance of a nursing laboratory.

UNITERMS: Nursing education. Teaching.

\section{REFERÊNCIAS BIBLIOGRÁFICAS}

ARBUÉS-MOREIRA, M.T.; FRIEDLANDER, M.R. A eficácia da cinta de video en la demonstracion de un procedimento de enfermeria. Aceito para publicaçáo na Rev. Enferm., 1992/no preio/

BALDWIN, D. et al Performance of psychomotor skills: a comparison of two teaching strategies. J.Nurs.Educ, v.30, n.8. p. 367-70. 1991.

BELL, M.L. Learning a complex nursing skill: student anxiety and the effect of preclinical skill evaluation. J.Nurs.Educ, v.30, n.5, p. 222-6, 1991 ,

BLENNER, J.L. Researcher for a day: a simulation game. Nurse Edux, v.16, n.2, p. 32-5. 1981.

BODY, S. et al Teaching injection technique: study of instrucional methods. A.A.R. Neydett, v.30, n.9, p.7-8, 1993.

BUTTERFIELD, S.H. In defense of the demonstration room. Int.NursRev., v.30, n.1, p. 15-20, 1983.

CORCORAN, S. Should a service retting be used as a learning laboratory? An ethical question. NursOutlook , v.25, a.11, p.771-6, 1977.

ELLIOTT, R. et al Psychomotor skill acquisicion. CanNurse v.78, n.3, p. 25-7, 1982. 
FRIEDLANDER, M.R. O ensino dos procedimentos breicos de enfermagem- comparação entre dois métodos de instrução. Såo Paulo, 1984. 142p. Tese(Doutorado)- Escola de Enfermagem, Universidade de Sảo Paulo.

FRIEDLANDER, M.R.; SOARES, J.M.S. A aprendizagem auto-dirigida no laboratorio de enfermagem/Apresentado no 45 Congresso Brasileiro de Enfermagem. Recife, 1993-bJ

FRIEDLANDER, M.R.; LUACUTE, J. O uso de roteiro auto-instrucionais no laboratório de enfermagem. Sảo Paulo, 1993c /mimeografado/.

GOMEZ.G.E.: GOMEZ. E.A. Learning of psychomotor skills: laboratory versus patients care setting. J.Nurs.Educ, v.26, n.1, p.20-4, 1987.

GOTHLER, A.M. Critical insues in nursing education: the clinical laboratory. J.N.Y.S.N.A. , v.16. n.3, p.51-5, 1985.

HALLAL, J.C.; WELSH, M.D. Using the competency laboratory to learn psychomotor skills. Nurse Educ,v.9, a.1, p. 34-8, 1984.

HAYASHIDA, M. Laboratorio de enfermagem: histórico organizacional e funcional de uma unıdade unıversitária. Ribeirão Preto, 1992. 119p. Dissertação(Mestrado)-Escola de Enfermagem de Ribeirảo Preto. Univeraidade de São Paulo.

HEGSTAD, L.N.; ZSOHAR. H.A. Study of the cost-effectiveness of providing psychomotor proctive in teaching intravenous infusion techniques. J.Nurs. Educ, v. 25, n.1, p. 10-4. 1986.

HODSON. K.E. et al. Integration of computerized assessment screens into psychomotor skill modules at o School of Nursing. Computwurs. v.6. a.3. p. 103-7. 1988.

INFANTE. M.S. Toward effective and efficient use of the clinical laboratory. Nurse Educ. v.6, n.1, p.16-9. 1981 .

Press. 1985.

The clinical laboratory in nursing education. New York. Assocsation

McDONALD. G.F. The sımulated clinical laboratory. Nurs.Outlook, v.35, n.6. p.290-2. 1987.

MEGEL, M.E. et al Nursing students performance: administering injection in laboratory and clinical area. J.Nurs. Educ.v.26, n.7, p.288-93, 1987.

VASCIMENTO, E.M.F.; CANÇADO, M.L.B. O uso de um sistema artesanalmente construido para o ensino prático da punçăo e infusāo intravenoso. /Apresentado no 43 Congresso Brasileiro de Enfermagem, Curitiba, 1991J

PAGANA. K.D. Stresses and threats reported by baccalaureate students in relation to an inicial clinical expenence. JNura.Educ, ,v.27, n.9. p.418-24, 1988.

TAYLOR, J.A.; CLEVELAND. P.J. Effective use of the learaing laboratory. J.Nurs.Educ., v.23, n.1, p.32-4, 1984. 